\title{
A seven-gene signature model predicts overall survival in kidney renal clear cell carcinoma
}

\author{
Ling Chen ${ }^{1}$, Zijin Xiang ${ }^{2}$, Xueru Chen ${ }^{2}$, Xiuting Zhu² and Xiangdong Peng ${ }^{2^{*}}$ (D)
}

\begin{abstract}
Background: Kidney renal clear cell carcinoma (KIRC) is a potentially fatal urogenital disease. It is a major cause of renal cell carcinoma and is often associated with late diagnosis and poor treatment outcomes. More evidence is emerging that genetic models can be used to predict the prognosis of KIRC. This study aimed to develop a model for predicting the overall survival of KIRC patients.

Results: We identified 333 differentially expressed genes (DEGs) between KIRC and normal tissues from the Gene Expression Omnibus (GEO) database. We randomly divided 591 cases from The Cancer Genome Atlas (TCGA) into training and internal testing sets. In the training set, we used univariate Cox regression analysis to retrieve the survival-related DEGs and futher used multivariate Cox regression with the LASSO penalty to identify potential prognostic genes. A seven-gene signature was identified that included APOLD1, C9orf66, G6PC, PPP1R1A, CNN1G, TIMP1, and TUBB2B. The seven-gene signature was evaluated in the training set, internal testing set, and external validation using data from the ICGC database. The Kaplan-Meier analysis showed that the high risk group had a significantly shorter overall survival time than the low risk group in the training, testing, and ICGC datasets. ROC analysis showed that the model had a high performance with an AUC of 0.738 in the training set, 0.706 in the internal testing set, and 0.656 in the ICGC external validation set.
\end{abstract}

Conclusion: Our findings show that a seven-gene signature can serve as an independent biomarker for predicting prognosis in KIRC patients.

Keywords: Kidney renal clear cell carcinoma, Bioinformatics, Prognostic model, LASSO penalty

\section{Background}

Kidney renal clear cell carcinoma (KIRC) is a type of renal cortical tumour characterized by a growth pattern of the cytoplasm that is associated with malignant epithelial cells and accounts for $80-90 \%$ of renal cell carcinomas. In addition, KIRC tends to be resistant to radiation and chemotherapy, which makes surgery the primary treatment [1]. However, $30 \%$ of patients who undergo surgery still experience metastasis [2]. Early

\footnotetext{
*Correspondence: xdpeng@csu.edu.cn

${ }^{2}$ Department of Pharmacy, The Third Xiangya Hospital, Central South University, Changsha 410013, Hunan, China

Full list of author information is available at the end of the article
}

identification of risk in KIRC patients can help with more accurate clinical treatment. Therefore, there is a strong demand to discover new and reliable markers to predict patient prognosis.

Many studies show that predictive models of gene expression have great significance in clinical prognosis applications. For example, Fatai et al. built a model to demonstrate that a 35-gene signature can discriminate between rapidly and slowly progressing glioblastoma multiforme and predict survival in known subtypes of cancer [3]. Long et al. constructed a prognostic model for patients with hepatocellular carcinoma based on RNA sequencing data [4]. For KIRC, Zhan et al. found 
that the expression of the five-gene model was related to the prognosis of patients with KIRC by Cox regression analysis [5]. Han et al. analysed reversed-phase protein array (RPPA) data for the protein expression signature of survival time in KIRC [6]. However, the studies of multigene models to predict the prognosis of KIRC patients are still insufficient, and we sought here to use a variety of methods to find more potentially relevant genes.

In terms of survival analysis, Cox proportional hazards regression is currently the most widely used method. However, it is not the most suitable method for highdimensional microarray data because overfitting is a common shortcoming of modelling using highdimensional microarray data to identify prognostic genes [7]. The LASSO method can eliminate this limitation and it was applied in our analysis for feature selection [8]. In this study, we sought to identify DEGs associated with OS based on genome-wide expression profiles of KIRC patients [9]. We developed a seven-gene signature by multivariate Cox proportional hazard regression with LASSO penalty $[10,11]$. The prognostic model involving these seven DEGs effectively divided KIRC patients into high- and low-risk groups; OS was significantly poorer in the high-risk group than in the low-risk group among the training, testing and ICGC sets. OS was regarded as the endpoint for evaluating the prognostic model and the ultimate measure of treatment benefits $[12,13]$. In conclusion, this study may add literature to existing prognostic models of KIRC to identify patients with a higher risk of mortality.

\section{Results}

\section{Screening for DEGs and GO enrichment analysis}

After GEO data filtering, quality assessment, and data processing, we performed differential expression analysis by using the limma $\mathrm{R}$ package and identified 333 DEGs from the GEO cohort.. These DEGs comprised 218 upregulated genes and 115 downregulated genes, using the criteria of $\log \mathrm{FC}>2$ or $\operatorname{logFC}<(-2)$ with adjusted $P<0.05$ (Fig. 1a). The heatmap in Fig. $1 \mathrm{~b}$ shows that the 333 DEGs were enriched in 4 nodes. GO analysis revealed that the DEGs were enriched in renal system development, kidney epithelium development, renal tubule development, and kidney development.

\section{Construction of a prognostic model in the training set}

DEGs that mediate tumour initiation, progression, and proliferation are potential prognostic biomarkers. To identify potential prognostic DEGs, the TCGA cohort was randomly divided into a training set $(n=300)$ and an internal testing dataset $(n=291)$ with an approximate ratio of 1:1. Consequently, a univariate Cox regression was first performed to filter out the DEGs that were not related to OS, and then 315 survival-related DEGs were identified. Based on the 315 survival-related DEGs, the relative regression coefficients were calculated by multivariate Cox regression with LASSO penalty. Using this method, we obtained seven potential prognostic genes, including APOLD1, C9orf66, G6PC, PPP1R1A, CNN1G, TIMP1, and TUBB2B (Fig. 2a; Table 1).

A risk score (RS) was calculated for each patient in the TCGA training set by combining the relative expression of the DEGs in the prognostic model and the LASSO coefficients. Patients with an RS $\geq 0.323$ (median cutoff) were classified as high risk and the remaining patients were classified as low risk, as shown in Fig. 2b. To investigate the relationship between RS and KIRC patients' OS, a Kaplan-Meier analysis and log-rank test were performed using the training set. We found that high-risk patients had a worse prognosis than low-risk patients (Fig. 2c). The area under the curve (AUC) value was 0.738 , as shown in the time-dependent receiver operating characteristic (ROC) curve assessing prognosis in Fig. 2d.

\section{Validation of the prognostic model using the TCGA and ICGC datasets}

To further explore the relationship between RS and KIRC patients' OS, a Kaplan-Meier analysis and logrank test were performed on the TCGA and ICGC validation sets. In the TCGA validation set, we used the same prognostic model; patients with an $\mathrm{RS} \geq 0.365$ were classified as high risk and the remaining patients were classified as low risk by using the median of all risk scores (Fig. 3a). It is clear that the OS was significantly lower for patients with a higher RS than for patients with a lower RS $(P<0.0001$; Fig. 3b). As most events occurred within 5 years, we used a time-dependent ROC curve to assess prognosis (Fig. 3c); the AUC value was 0.706 . To verify that our prognostic model can be applied universally, we further applied the seven-gene signature to ICGC data. A total of 159 samples were obtained from the ICGC database, and after batch effect, 157 samples remained. Using the median cutoff of $\mathrm{RS}=$ 0.644 (Fig. $3 \mathrm{~d}$ ), the prognostic model successfully subdivided the patients into a high-risk group or a lowrisk group, and the OS was significantly different. The five-year survival rate of patients in the high-risk group was low (Fig. 3e). The time-dependent ROC curve demonstrated an AUC of 0.656 (Fig. 3f), which showed better prediction performance. Moreover, we demonstrated the universal prognostic value of the seven-gene signature in the TCGA cohort despite the pathological stage, especially for stages I, III and IV (all $P<0.05$, Table 2). 


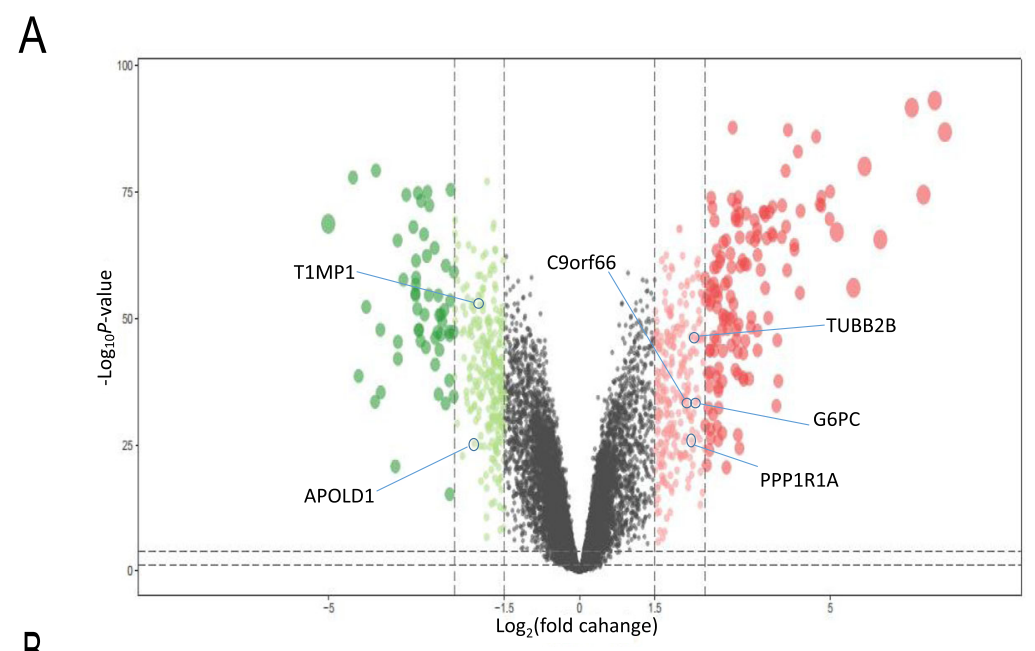

B

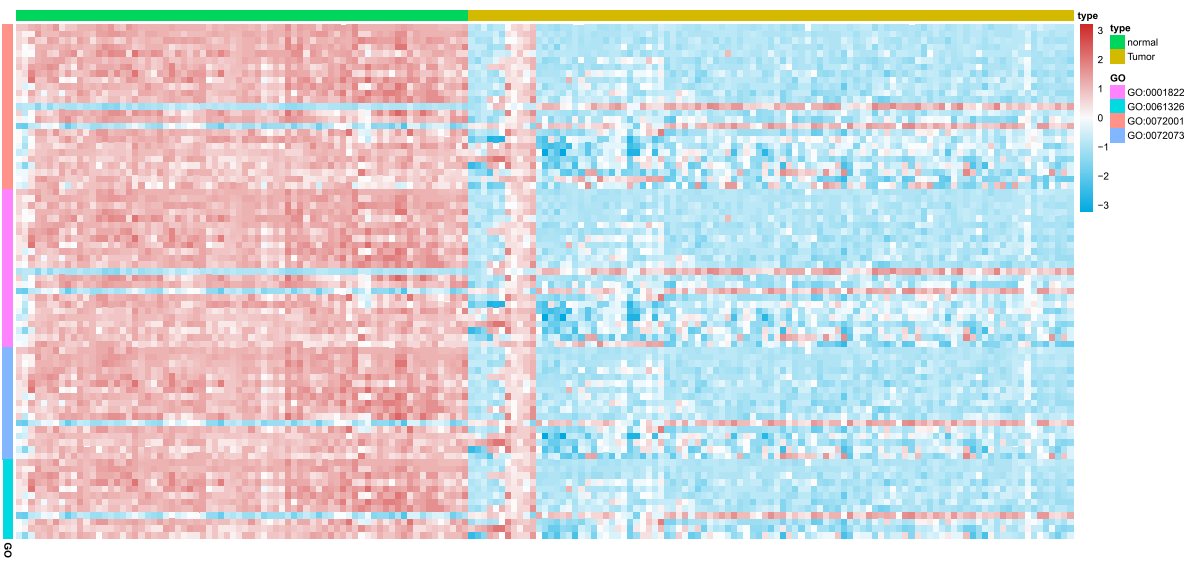

Fig. 1 DEGs in KIRC vs adjacent normal tissues. a. Volcano plot visualizing the DEGs screened using limma. The red and green points represent the significantly upregulated and downregulated DEGs, respectively (logFC $>2$ or $\log \mathrm{FC}<(-2)$ with adjusted $P<0.05$ ). Features selected by the LASSO penalty are also marked. b. Heatmap showing that the 333 DEGs are involved in renal system development, kidney epithelium development, renal tubule development, and kidney development

\section{Developing and validating a predictive nomogram based on the seven-gene prognostic model}

To establish a survival prediction method for KIRC patients, a nomogram was used to predict the probability of three- and five-year OS in the TCGA cohort. The predictors in the nomogram included four independent prognostic factors (age, gender, tumour stage, and race (Fig. 4a) [14]. The calibration curve illustrated that the predictions and actual observations matched well, which indicated an accurate prediction via the nomogram (Fig. 4b) [15].

\section{Discussion}

There is growing evidence that, despite the importance of individual molecules, tumorigenesis and prognosis are strictly controlled by interactions between a large number of cellular components including DNA, RNA, proteins, and small molecules [16]. However, the number of specific biomarkers with prognostic significance is still small [17], and the identification of prognostic factors is important for the optimal treatment of KIRC patients. Therefore, to reduce mortality and improve the prognosis of KIRC, molecular screening of KIRC biomarkers is urgently needed. In this study, we identified 333 DEGs by analysing GEO data. We then conducted a GO enrichment analysis, showing that the 333 DEGs are primarily involved in renal system development, kidney epithelium development, renal tubule development, and kidney development. After multivariate Cox regression with LASSO penalty, seven DEGs were identified, and two validation analyses were performed using independent datasets, showing good reproducibility.

The biological functions of the seven identified DEGs have been reported in previous studies. However, only a few of the DEGs have been investigated in KIRC. APOLD1 (Apolipoprotein L Domain Containing 1) is an 


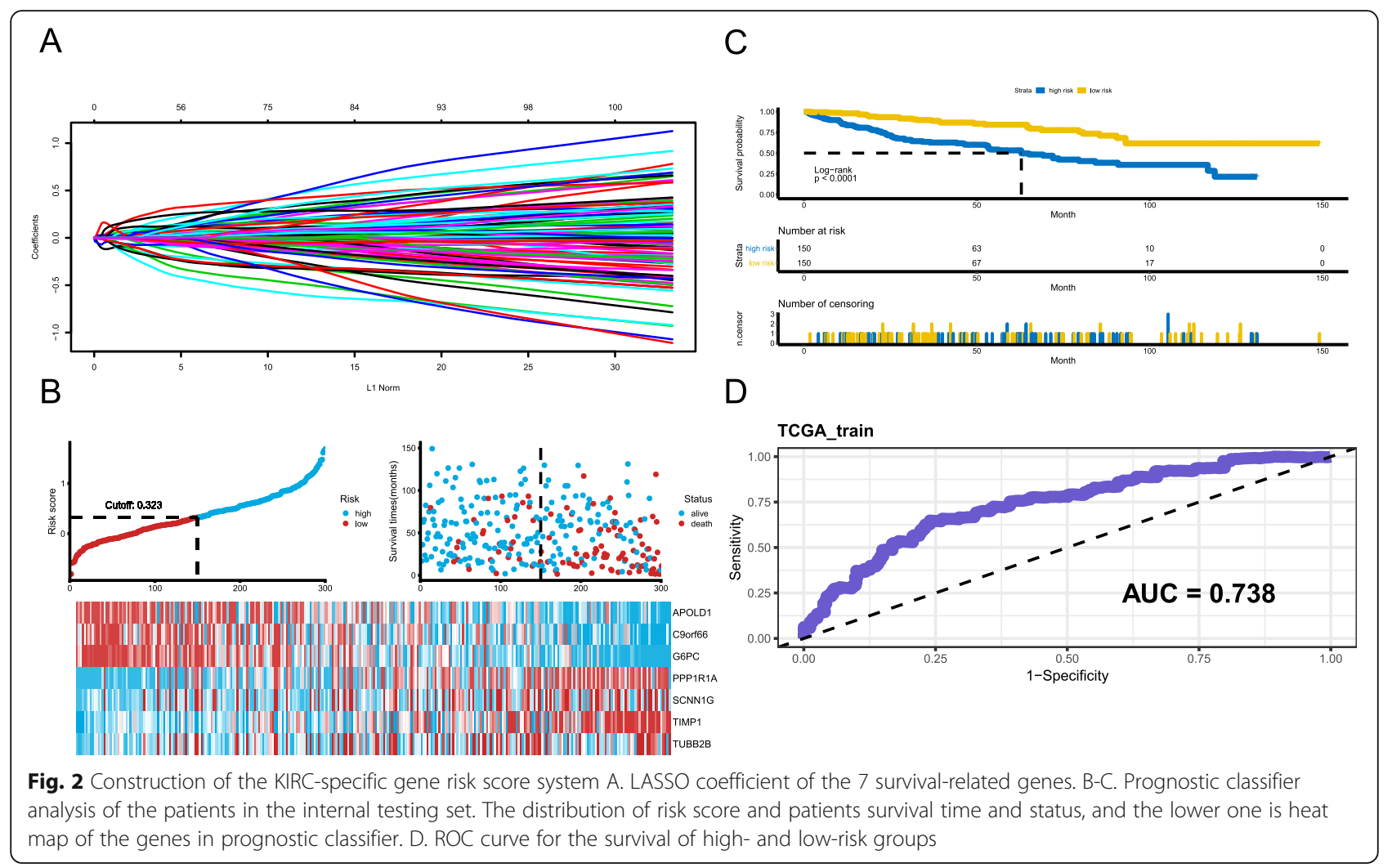

endothelial cell early response protein that may play an important role in the regulation of endothelial signalling pathways and vascular function. C9orf66 (Chromosome 9 Open Reading Frame 66) is a protein-coding gene. G6PC (Glucose-6-Phosphatase Catalytic Subunit) is also a protein-coding gene. Any defects in this gene abrogate G6Pase function [18-20], which is associated with increased glycogen accumulation in gluconeogenic organs, especially in the kidneys, where it promotes progressive nephromegaly [21]. Poor metabolic control often results in long term complications such as renal dysfunction, pancreatitis, and hypertriglyceridemia, impairing kidney function and increasing the probability of KIRC [21]. PPP1R1A (Protein Phosphatase 1 Regulatory Inhibitor Subunit 1A) is a protein-coding gene [22]. TIMP1
(TIMP Metallopeptidase Inhibitor 1) is also a proteincoding gene [23]. The proteins encoded by this gene family are natural inhibitors of matrix metalloproteinases (MMPs). In addition to its inhibitory role against most of the known MMPs, TIMP1 promotes cell proliferation in a wide range of cell types and may also have an antiapoptotic function. TUBB2B (Tubulin Beta 2B Class IIb) is a protein-coding gene. TUBB2B mutation leads to tubulin heterodimerization impairment, decreased ability to incorporate into the cytoskeleton, and alteration of microtubule dynamics, with an accelerated rate of depolymerization, which causes renal disease and an increase in the incidence of KIRC [24].

Compared to previous research, our study had some differences [25, 26]. First, our risk score (RS) strategy

Table 1 Details of features selected by multivariate Cox proportional hazard regression model with LASSO penalty

\begin{tabular}{llc}
\hline Gene Name & \multicolumn{1}{c}{ Description } & LASSO coefficient \\
\hline APOLD1 & Apolipoprotein L Domain Containing 1 & -0.09978 \\
C9orf66 & Chromosome 9 Open Reading Frame 66 & -0.01573 \\
G6PC & Glucose-6-Phosphatase Catalytic Subunit & -0.06969 \\
PPP1R1A & Protein Phosphatase 1 Regulatory Inhibitor Subunit 1A & 0.02551 \\
SCNN1G & Sodium Channel Epithelial 1 Subunit Gamma & 0.01383 \\
TIMP1 & TIMP Metallopeptidase Inhibitor 1 & 0.13582 \\
TUBB2B & Tubulin Beta 2B Class Ilb & 0.02215 \\
\hline
\end{tabular}




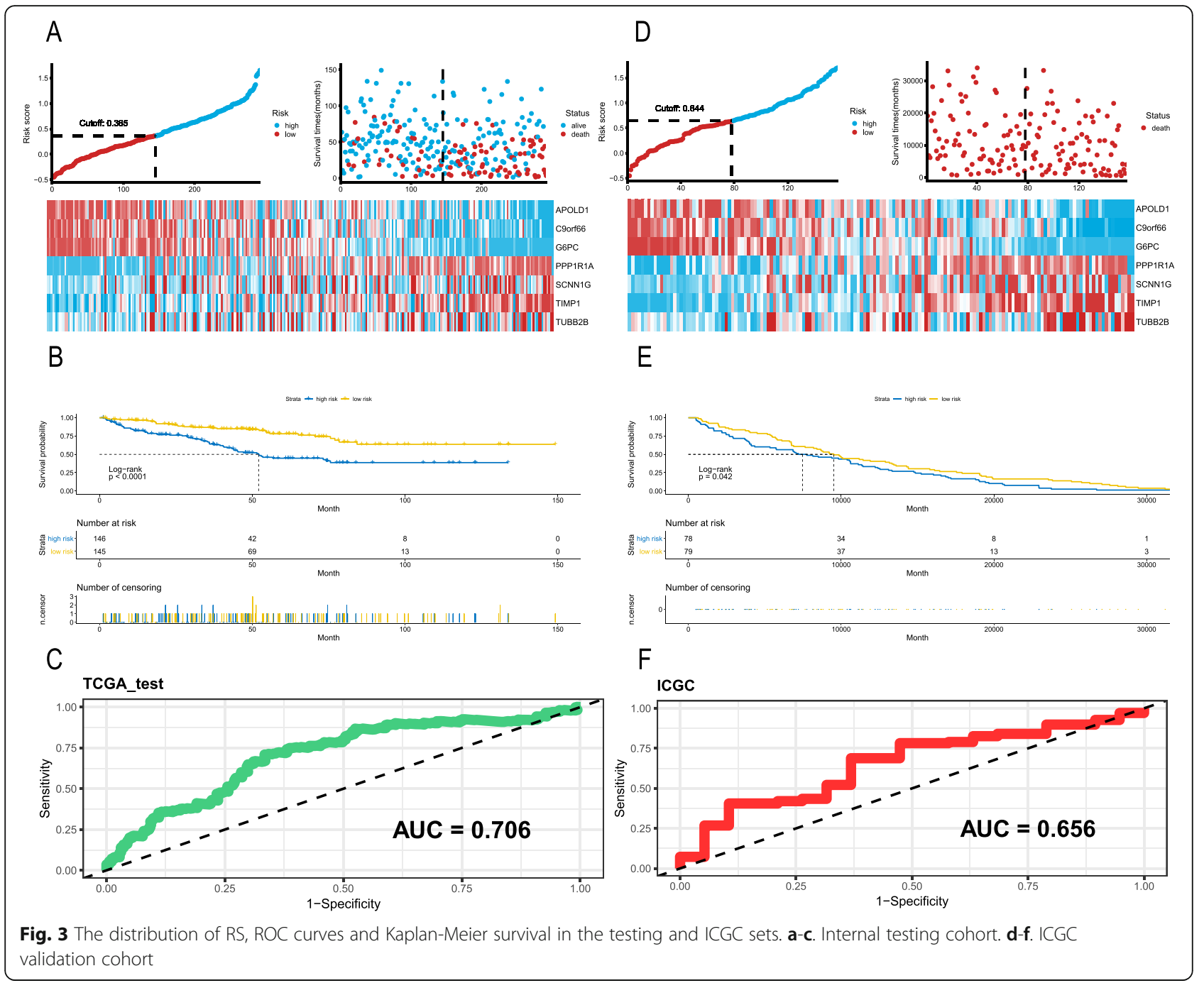

involved LASSO penalized regression which can analyse all independent variables as well as the most influential variables. When dealing with large datasets such as gene expression profiles, this method is much more accurate than the stepwise regression method of multivariate Cox regression models. Moreover, we used data from GSE8050, GSE12606, GSE14762, GSE36895, and GSE46699 KIRC expression profiling chips to identify DEGs and TCGA data for validation, and we then used ICGC data for external validation. We also acknowledge the limitations of this study. First, before clinical application, PCR-based sample validation should be conducted.
Second, the functional phenotypes and mechanisms of the seven genes deserve further investigation. Third, a treatment effect that would influence patients' prognosis was ignored when developing a prognostic model due to incomplete medical records.

\section{Conclusion}

In summary, we developed a seven-gene signature that is associated with OS in KIRC patients. Our findings suggest that the seven-gene signature can serve as an independent biomarker for predicting survival prognosis, and we are poised for further investigation and eagerly

Table 2 Prognostic value of 7-gene signature in different pathological stages of KIRC in the TCGA cohort

\begin{tabular}{|c|c|c|c|c|c|}
\hline & Number of samples & Number of Death (\%) & Hazard Ratio & $95 \% \mathrm{Cl}$ & $\boldsymbol{P}$ Value \\
\hline Stage I & 214 & $35(16)$ & 5.16 & $2.07-12.87$ & 0.0004 \\
\hline Stage ॥ & 42 & $7(17)$ & 1.15 & $0.11-11.54$ & 0.908 \\
\hline Stage III & 116 & $46(40)$ & 2.12 & $1.05-4.27$ & 0.037 \\
\hline Stage IV & 71 & $56(79)$ & 3.73 & $1.67-8.32$ & 0.001 \\
\hline
\end{tabular}



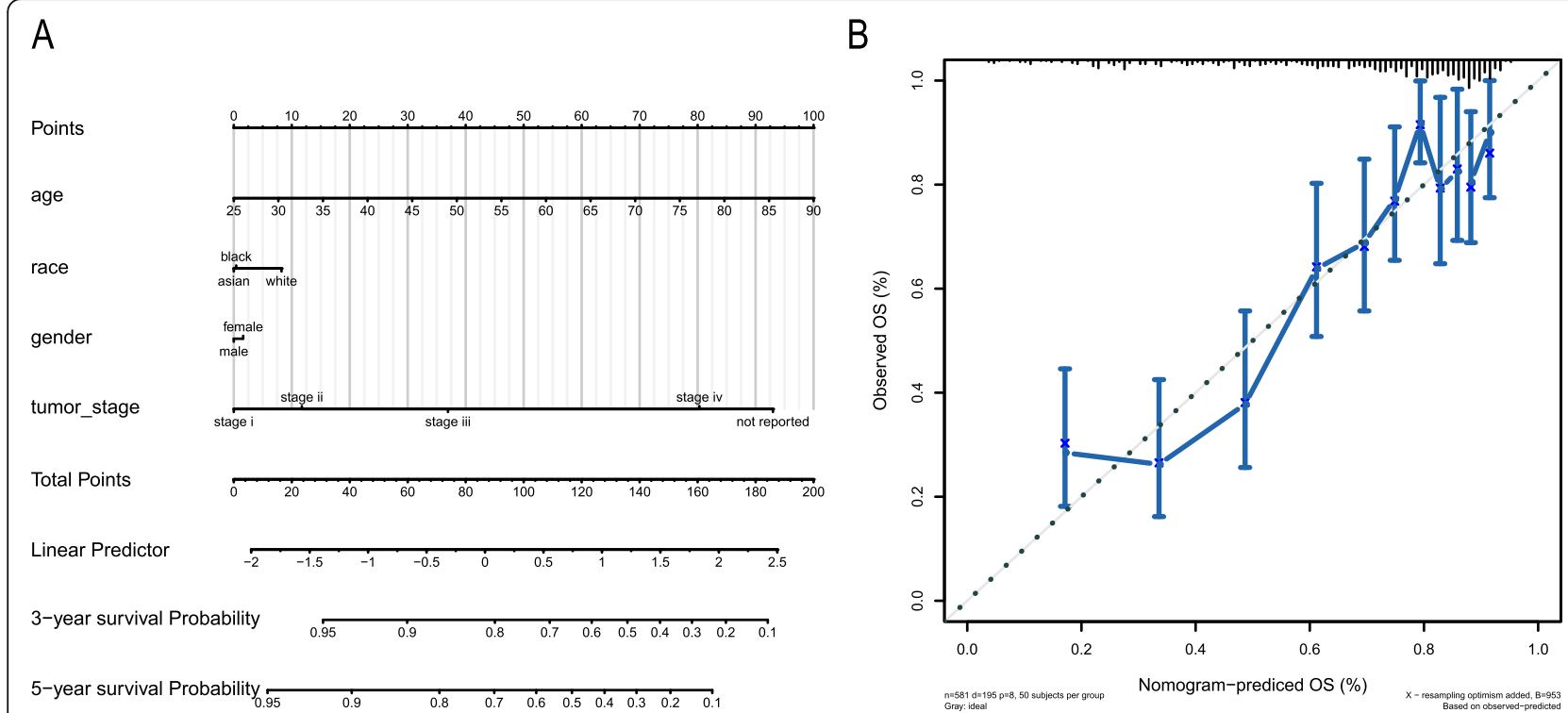

Fig. 4 Nomogram for predicting 3- and 5-year OS. a. We added up the points identified on the points scale for each variable that can be projected onto the scales to indicate the probability of 3- and 5-year OS. b. Calibration plot showing the prediction of OS. The nomogrampredicted probability of OS is plotted on the x-axis; actual OS is plotted on the $y$-axis

anticipate the verification of our findings in a larger cohort of patients to assess whether the seven genes are likely to become new drug treatment targets.

\section{Methods}

\section{KIRC sample sources}

The following five KIRC expression profiling chip datasets, based on the GPL570 platform, were downloaded from the Gene Expression Omnibus (GEO) database: GSE8050, GSE12606, GSE14762, GSE36895, and GSE46699 with a total of 218 KIRC and normal kidney tissue samples. After removal of the samples with inadequate clinical information, 99 KIRC and 74 normal control samples were selected for this analysis. KIRC clinical and gene expression data (605 cases) were downloaded from the TCGA database, and a total of 591 cases ware obtained after removing the batch effect. This study strictly followed the published guidelines issued by TCGA. The TCGA data were randomly divided and used as a prognostic model training set and an internal testing set, and the ICGC data were used as an external validation set.

\section{Screening for differentially expressed genes (DEGs)}

Differentially expressed genes (DEGs) were identified by $\mathrm{R}$ software and the screening criteria were absolute logFoldChange $>2$ with adjusted $P<0.05$. A total of 333 DEGs were identified between 99 KIRC and 74 normal control samples. These genes were then mapped to the TCGA and International Cancer Genome Consortium
(ICGC) databases using the ID database. Excluding unmatched genes, 315 genes were available for analysis.

\section{Gene ontology (GO) enrichment analysis of DEGs}

The biological significance of the DEGs was explored using a GO term enrichment analysis of biological processes, cellular components, and molecular functions. The search tool for recurring instances of neighbouring genes (STRING) [27] was used by inputting the gene name of each DEG and exporting the results [28].

\section{Screening for KIRC survival-related genes}

We randomly divided the 591 TCGA samples with approximate ratio of $1: 1$ and 300 samples were set as the training set and 291 samples were set as the internal testing set. In the training group, multivariate Cox proportional hazard regression analysis was performed on 315 DEGs [29, 30], followed by LASSO penalty to further screen out a group of independent prognostic candidate genes with the strongest predictive power [31].

\section{Survival analysis}

All statistical analyses were conducted by R3.6.2. Kaplan-Meier curves were generated for survival rates of patients, with difference detection based on log-rank testing. A Cox proportional hazard regression model was used to calculate the hazard ratios (HRs) and 95\% confidence intervals (CIs) regarding OS [13]. Specifically, survival curves were established in the training set, internal testing set and ICGC set. The predictive performance of the nomogram was evaluated by a calibration 
curve [15]. For all statistical analyses, a two-tailed $P$ value less than 0.05 was considered statistically significant.

\section{Abbreviations}

KIRC: Kidney renal clear cell carcinoma; DEGs: Differentially expressed genes; GEO: Gene Expression Omnibus; TCGA: The Cancer Genome Atlas; ICGC: International Cancer Genome Consortium; OS: Overall survival; LASSO: Least absolute shrinkage and selection operator; ROC: Receiveroperator characteristic; AUC: Area under curve; STRING: Search Tool for Recurring Instances of Neighbouring Genes; RS: Risk score; RPPA: Reverse phase protein array; APOLD1: Apolipoprotein L Domain Containing 1 C9orf66: Chromosome 9 Open Reading Frame 66; G6PC: Glucose-6Phosphatase Catalytic Subunit; PPP1R1A: Protein Phosphatase 1 Regulatory Inhibitor Subunit 1A; TIMP1: TIMP Metallopeptidase Inhibitor 1; MMPs: matrix metalloproteinases; TUBB2B: Tubulin Beta 2B Class Ilb

\section{Acknowledgements}

We greatly appreciate the patients and investigators who participated in TCGA and GEO for providing data. The results published or shown here are in whole or part based upon data generated by the TCGA Research Network: https://www.cancer.gov/tcga.

\section{Authors' contributions}

All authors participate in data collection, data analysis and manuscript preparation. The authors read and approved the final manuscript.

\section{Funding}

This study was supported by Hunan Provincial Natural Science Foundation of China (2018JJ2600), Key R\&D Program of Hunan Province (China) NO.2018SK2129, Project of Scientific Research Plan of Hunan Provincial Health Commission B2017029.

\section{Availability of data and materials}

All analyzed data related to this paper are included in this paper.

\section{Ethics approval and consent to participate}

Not applicable.

\section{Consent for publication}

All the authors have consented for the publication.

\section{Competing interests}

The authors declare that they have no competing interests.

\section{Author details}

'Department of Gastrointestinal Surgery, Xiangya Hospital, Central South University, Changsha 410013, Hunan, China. ${ }^{2}$ Department of Pharmacy, The Third Xiangya Hospital, Central South University, Changsha 410013, Hunan, China.

Received: 28 March 2020 Accepted: 26 August 2020

Published online: 03 September 2020

\section{References}

1. Hsieh JJ, Purdue MP, Signoretti S, Swanton C, Albiges L, Schmidinger M, et al. Renal cell carcinoma. Nat Rev Dis Primers. 2017;3:17009.

2. Motzer RJ, Hutson TE, Cella D, Reeves J, Hawkins R, Guo J, et al. Pazopanib versus sunitinib in metastatic renal-cell carcinoma. N Engl J Med. 2013; 369(8):722-31.

3. Fatai AA, Gamieldien J. A 35-gene signature discriminates between rapidlyand slowly-progressing glioblastoma multiforme and predicts survival in known subtypes of the cancer. BMC Cancer. 2018;18(1):377.

4. Long J, Zhang L, Wan X, Lin J, Bai Y, Xu W, et al. A four-gene-based prognostic model predicts overall survival in patients with hepatocellular carcinoma. J Cell Mol Med. 2018;22(12):5928-38.

5. Zhan Y, Guo W, Zhang Y, Wang Q, Xu XJ, Zhu L. A five-gene signature predicts prognosis in patients with kidney renal clear cell carcinoma. Comput Math Methods Med. 2015;2015:842784.

6. Han G, Zhao W, Song X, Kwok-Shing Ng P, Karam JA, Jonasch E, et al. Unique protein expression signatures of survival time in kidney renal clear cell carcinoma through a pan-cancer screening. BMC Genomics. 2017; 18(Suppl 6):678.

7. Wu TT, Chen YF, Hastie T, Sobel E, Lange K. Genome-wide association analysis by lasso penalized logistic regression. Bioinformatics. 2009;25(6): 714-21.

8. Oster $\mathrm{B}$, Linnet $\mathrm{L}$, Christensen $\mathrm{L} L$, Thorsen $\mathrm{K}$, Ongen $\mathrm{H}$, Dermitzakis ET, et al. Non-CpG island promoter hypomethylation and miR-149 regulate the expression of SRPX2 in colorectal cancer. Int J Cancer. 2013;132(10): $2303-15$

9. Liu C, Wang X, Genchev GZ, Lu H. Multi-omics facilitated variable selection in cox-regression model for cancer prognosis prediction. Methods. 2017;124: $100-7$.

10. Henriques J, Pujades-Rodriguez M, McGuire M, Szumilin E, Iwaz J, Etard JF, et al. Comparison of methods to correct survival estimates and survival regression analysis on a large HIV African cohort. PLoS One. 2012;7(2): e31706

11. Tang Z, Shen $Y$, Zhang $X$, Yi N. The spike-and-slab lasso cox model for survival prediction and associated genes detection. Bioinformatics. 2017; 33(18):2799-807.

12. Barrett T, Troup DB, Wilhite SE, Ledoux P, Rudnev D, Evangelista C, et al. NCBI GEO: mining tens of millions of expression profiles--database and tools update. Nucleic Acids Res. 2007;35(Database issue):D760-5.

13. George B, Seals S, Aban I. Survival analysis and regression models. J Nucl Cardiol. 2014;21(4):686-94

14. Jacob H, Stanisavljevic L, Storli KE, Hestetun KE, Dahl O, Myklebust MP. A four-microRNA classifier as a novel prognostic marker for tumor recurrence in stage II colon cancer. Sci Rep. 2018;8(1):6157.

15. Zhao H, Cao Y, Wang Y, Zhang L, Chen C, Wang Y, et al. Dynamic prognostic model for kidney renal clear cell carcinoma (KIRC) patients by combining clinical and genetic information. Sci Rep. 2018;8(1):17613.

16. Song M. Recent developments in small molecule therapies for renal cell carcinoma. Eur J Med Chem. 2017:142:383-92.

17. Mei S, Li F, Leier A, Marquez-Lago TT, Giam K, Croft NP, et al. A comprehensive review and performance evaluation of bioinformatics tools for HLA class I peptide-binding prediction. Brief Bioinform. 2020;21(4):1119-1135.

18. Aiston S, Trinh KY, Lange AJ, Newgard CB, Agius L. Glucose-6-phosphatase overexpression lowers glucose 6-phosphate and inhibits glycogen synthesis and glycolysis in hepatocytes without affecting glucokinase translocation. Evidence against feedback inhibition of glucokinase. J Biol Chem. 1999; 274(35):24559-66.

19. Ichai C, Guignot L, El-Mir MY, Nogueira V, Guigas B, Chauvin C, et al. Glucose 6-phosphate hydrolysis is activated by glucagon in a low temperaturesensitive manner. J Biol Chem. 2001:276(30):28126-33.

20. Roseman DS, Khan T, Rajas F, Jun LS, Asrani KH, Isaacs C, et al. G6PC mRNA therapy positively regulates fasting blood glucose and decreases liver abnormalities in a mouse model of glycogen storage disease 1a. Mol Ther. 2018:26(3):814-21

21. Chou JY, Mansfield BC. Mutations in the glucose-6-phosphatase-alpha (G6PC) gene that cause type la glycogen storage disease. Hum Mutat. 2008; 29(7):921-30.

22. Jiang L, Brackeva B, Ling Z, Kramer G, Aerts JM, Schuit F, et al. Potential of protein phosphatase inhibitor 1 as biomarker of pancreatic beta-cell injury in vitro and in vivo. Diabetes. 2013;62(8):2683-8.

23. Jaglin XH, Poirier K, Saillour Y, Buhler E, Tian G, Bahi-Buisson N, et al Mutations in the beta-tubulin gene TUBB2B result in asymmetrical polymicrogyria. Nat Genet. 2009;41(6):746-52.

24. Jeruschke $S$, Jeruschke $K$, DiStasio A, Karaterzi S, Büscher AK, Nalbant $P$, et al. Everolimus stabilizes Podocyte microtubules via enhancing TUBB2B and DCDC2 expression. PLoS One. 2015;10(9):e0137043.

25. Dimitrieva S, Schlapbach R, Rehrauer H. Prognostic value of cross-omics screening for kidney clear cell renal cancer survival. Biol Direct. 2016;11(1):68.

26. Song J, Liu YD, Su J, Yuan D, Sun F, Zhu J. Systematic analysis of alternative splicing signature unveils prognostic predictor for kidney renal clear cell carcinoma. J Cell Physiol. 2019:234(12):22753-64

27. Szklarczyk D, Franceschini A, Kuhn M, Simonovic M, Roth A, Minguez P, et al. The STRING database in 2011: functional interaction networks of proteins, globally integrated and scored. Nucleic Acids Res. 2011; 39(Database issue):D561-8.

28. van Dijk PC, Jager KJ, Zwinderman AH, Zoccali C, Dekker FW. The analysis of survival data in nephrology: basic concepts and methods of cox regression. Kidney Int. 2008;74(6):705-9. 
29. Nicolai P. Redaelli de Zinis LO, Tomenzoli D, Barezzani MG, Bertoni F, Bignardi $\mathrm{M}$, et al. Prognostic determinants in supraglottic carcinoma: univariate and Cox regression analysis. Head Neck. 1997;19(4):323-34.

30. Ternes N, Rotolo F, Michiels S. Empirical extensions of the lasso penalty to reduce the false discovery rate in high-dimensional cox regression models. Stat Med. 2016;35(15):2561-73.

31. Frost $\mathrm{HR}$, Amos $\mathrm{Cl}$. Gene set selection via LASSO penalized regression (SLPR). Nucleic Acids Res. 2017;45(12):e114.

\section{Publisher's Note}

Springer Nature remains neutral with regard to jurisdictional claims in published maps and institutional affiliations.

Ready to submit your research? Choose BMC and benefit from:

- fast, convenient online submission

- thorough peer review by experienced researchers in your field

- rapid publication on acceptance

- support for research data, including large and complex data types

- gold Open Access which fosters wider collaboration and increased citations

- maximum visibility for your research: over $100 \mathrm{M}$ website views per year

At $\mathrm{BMC}$, research is always in progress.

Learn more biomedcentral.com/submissions 